\title{
Study on the Distinguish Method to Ensure Countermeasures of Traffic Hidden Trouble Points in Extreme Weather
}

\author{
SUI Liying \\ Senior engineer, Beijing Transportation Information \\ Center \\ Beijing, China \\ e-mail: suiliying@bjjtw.gov.cn \\ CHEN Zhihong \\ Deputy director, Transport Operation Coordinate \\ Center(TOCC) \\ Beijing, China \\ email: chenzhihong@bjjtw.gov.cn
}

\author{
NI Shunjiang \\ Instituteof Public Safety Research, Tsinghua University, \\ Beijing, China \\ email:sjni@tsinghua.edu.cn
}

\begin{abstract}
In recent years, extreme weather phenomenon has happened frequently, for example, rainstorm, hard snow, fog etc. At the same time, extreme weather phenomenon causes easily stagnant water, surface snow, ice on road, highway landslide and other disasters, which causes many traffic hazard points, brings huge influence to traffic safety. ${ }^{[1-2]}$ Basing on the existing accident, hidden trouble and a lot of monitoring data, using the fault tree analysis, historical case statistics and other methods to distinguish the traffic hazard points caused by extreme weather as accurately as possible, building a series of security countermeasures of traffic hidden trouble point in extreme weather, in order to realize the traffic emergency management sooner and serve for traffic security and people travel better ${ }^{[3-5]}$.

Key words: extreme weather; traffic; hidden points;
\end{abstract} distinguish; countermeasures

\section{INTRODUCTION}

In recent years, with the frequent occurrence of extreme weather, more and more traffic hidden trouble caused by extreme weather, hidden points with the feature of short time and uneven distribution, have brought great impact on traffic safety. How to identify the traffic hidden trouble points in extreme weather conditions quickly and accurately, and to formulate effective security countermeasures, has very important practical significance $^{[6-7]}$.

Traffic hidden danger recognition of this paper refers to identification persons monitor the transportation network environment by corresponding measures (such as technical testing, inspection on-site, etc.) , collect, analyze and evaluate all kinds of traffic operation index, search the regulations of violating relevant laws, regulations, rules, standards, regulations and safety production management system, or the factors which may cause the accident behavior, as accurately as possible to identify traffic danger points, namely search all kinds of risk factors.

\section{PRINCIPLE OF SELECTING THE TRAFFIC HIDDEN TROUBLE POINT IDENTIFICATION METHOD}

\section{A. Scientific principle}

Analysis of the existing hidden danger recognition work is to determine the risks in the system. The identification of traffic hidden trouble points in extreme weather conditions involves many aspects, in the actual work of hazard identification, should follow the scientific principles, recognize the hidden dangers from the road basic situation, road management situation, extreme weather factors etc., and make the recognition plan of traffic hidden trouble.

\section{B. Operation principle}

The identification methods of traffic hidden trouble should be based on the traffic industry characteristics, combined with the actual situation of the hidden danger recognition mechanism, choose feasible hidden identification methods, to ensure that this method plays a role in the actual danger points recognition. The operation of the identification method of danger points should be very strong, and constantly improve the process in practice.

\section{Systematic principle}

Traffic hidden trouble in extreme weather conditions and the whole traffic system are closely linked; a hidden danger may have direct or indirect relation with multiple factors. Therefore, in the danger points recognition, the system should be analyzed comprehensive and in detail, to study the relationship between the various parts of the system, analysis of the intrinsic link of each factor, analysis of hidden danger not only from the single factor, otherwise hidden danger recognition work is easy to become a mere form, and the recognition of work is not in depth, which may lead to the occurrence of the later 
accident.

\section{Comprehensive principle}

According to the systematic principle, we know that the traffic hidden troubles are caused by all factors of system internal. Therefore, in the hidden danger recognition work, we should analyze comprehensively each factor of the system, shouldn't be put in fluky psychology, so as to identify the various risks at all, to ensure the safety of the transportation system.

\section{IDENTIFICATION METHOD AND SELECTION \\ OF TRAFFIC HIDDEN TROUBLE IN EXTREME WEATHER CONDITIONS}

Extreme weather is likely to cause traffic hidden trouble, based on the theoretical methods of recognizing risks hidden trouble, combined with its own characteristics of traffic hidden trouble caused by extreme weather, this paper gives the key method which is suitable for traffic hidden trouble point recognition in extreme weather conditions, including fault tree analysis and historical case statistics ${ }^{[8-9]}$.

\section{A. Identification Method of Traffic Hidden Trouble in Extreme Weather Conditions}

Traffic hidden trouble in extreme weather conditions is one of the risks, so the recognition of traffic hidden trouble in extreme weather conditions can reference a lot of theory methods of risk hazard identification. Combining with its own characteristics of traffic hidden trouble caused by extreme weather, this paper analyzes the following recognition methods of traffic hidden trouble points in extreme weather, and compares and classifies according to the different weather types.

1)Fault tree analysis

Fault tree analysis is a process of logical analysis, descripting accident causation, and is the "tree" with direction, which is one of the important analysis methods of system safety engineering. It can identify and evaluate the risk of various systems, the qualitative and quantitative analysis of accident. Fault tree analysis is including event symbol and logic gate symbol. Among them, events include:

The top event: which is to be analyzed, generally accidents possible occur or have occurred as the top event, represented by the letter $\mathrm{T}$.

Intermediate event: refers to cause the top event events in the system, represented by the letter A(i).

The underlying event: the underlying event refers to events in the bottom layer of the fault tree, mainly include the basic events, the omission of events and house shaped events, these three kinds of bottom event unified using the letters X (I) said.

The basic events: these are one of the most basic events in system, say the most fundamental reason of the failure, and can't be analyzed further, represented by circular symbols.

The omission events: which are not need to be analyzed further, and can be used to represent errors events of such as drivers, represented by the diamond symbols.

House shaped events: said that the normal events to occur, the system in normal condition inevitable event, and that is not in need of repair failure, with house shaped symbol.

Logic gate symbols are used to connecting two or more events in the fault tree, the main logical relation can be transformed into "and" and "or". Or: as long as there is a place among the connected events $\mathrm{X} 1, \mathrm{X} 2 \ldots \mathrm{Xn}$, the event will occurs. And: the connected events Xl, X2 ... Xn are Simultaneous, the event will occur.

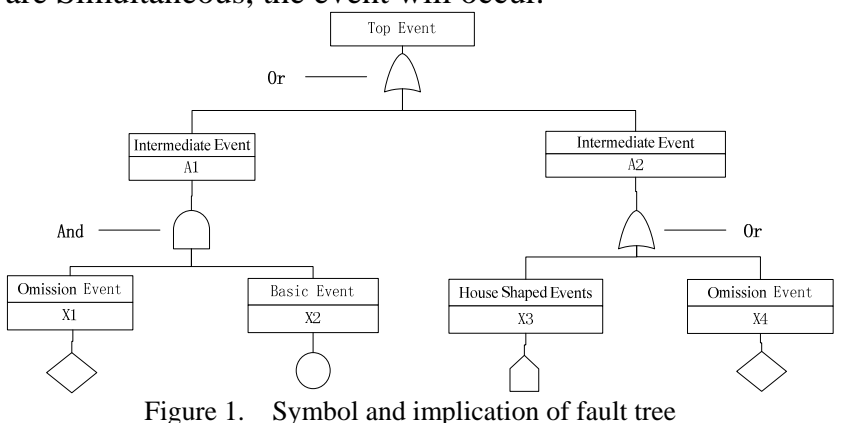

\section{2) Historical case statistics}

Through analyzing the various traffic related accident cases, finding out the occurrence rules of extreme weather conditions, such as the weather conditions when accident occurs, occurring sites and surrounding the situation etc., judging comprehensively the situation of the relevant sections, and then determines the risk, this method requires the collection of historical cases must be comprehensive and specific, in this way, analyzes the specific distribution of risks according to the accident data. This requires data should be specific, not careless.

\section{3)Check list method}

In order to identify the unsafe factors in the system, the system were analyzed in this paper, list the unsafe factors of all levels, then determine the examination items, which are organized a table in order to carry out the inspection or review, this is the check list.

\section{4)Model forecasting method}

The model forecast method is mainly through the hidden trouble data, and analyzes the risk of the possible occurrence. It can be generally used to establish the risk assessment model by risk factors, and other models can be set up by using some mathematical methods, such as gray forecasting model.

\section{5)Brainstorming method}

When group decision making, focusing related experts and organizing a special meeting, the host clarifies the issues in a clear way to all participants, and shows the meeting rules, tries to create a relaxed and harmonious atmosphere of the meeting. The host doesn't make comments generally, so as not to affect the freedom atmosphere of the conference. The experts may put forward the programs as many as possible.

\section{6)Monitoring and controlling method}

According to the daily monitoring data, real-time monitoring, analyzing evaluation object, and real time to know the latest situation, and take corresponding measures.

\section{B. Selection of Identification Method for Traffic Hidden Trouble Spots in Extreme Weather Conditions}

For the traffic risk identification caused of rain, snow, fog and other different extreme weather conditions should use different identification methods. The identification methods are shown the following table. 
TABLE 1. IDENTIFICATION METHOD OF TRAFFIC HAZARD IN DIFFERENT EXTREME WEATHER CONDITIONS

\begin{tabular}{|c|l|}
\hline $\begin{array}{c}\text { Extreme } \\
\text { Weather }\end{array}$ & \multicolumn{1}{c|}{ Hidden trouble identification method } \\
\hline Rain & $\begin{array}{l}\text { Fault tree analysis method, Brainstorming method, } \\
\text { Check list method, Historical case statistics, Model } \\
\text { forecasting method, Monitoring and controlling } \\
\text { method }\end{array}$ \\
\hline Snow & $\begin{array}{l}\text { Brainstorming method, Check list method, Historical } \\
\text { case statistics, Monitoring and controlling method }\end{array}$ \\
\hline Fog & $\begin{array}{l}\text { Brainstorming method, Historical case statistics, } \\
\text { Monitoring and controlling method }\end{array}$ \\
\hline
\end{tabular}

\section{APPLICATION OF TRAFFIC HIDDEN}

TROUBLE SPOTS IDENTIFICATION METHOD IN EXTREME WEATHER CONDITIONS

A certain national road K53.9-K54.7 section in Beijing is a typical accident prone section. According to incomplete statistics, more than 50 accidents had happened in 2004. Among them, 83\% of the accidents occurred in the rain. The main causes of accidents are related to adverse weather conditions, fatigue driving, speeding, overloading and vehicle rollover ${ }^{[10-11]}$. Together with some drivers' careless or disregarded, increasing the chance of accidents. Analyzing the accident data of this road section, the paper establishes the following fault tree model, which is as shown in the picture.

\section{Accident prone point analysis}

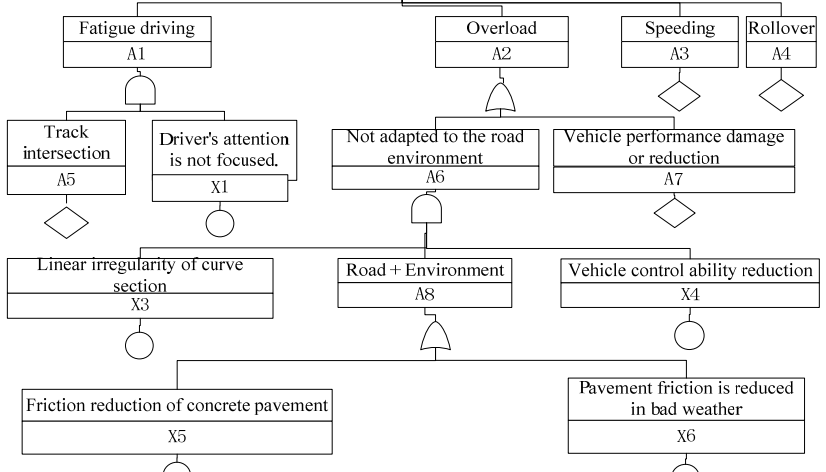

Figure 2. Fault prone tree analysis

\section{THE SAFEGUARD CONDITION OF TRAFFIC HAZARD IDENTIFICATION IN EXTREME WEATHER CONDITIONS}

A. Establish and Improve the Relevant Management System

B. Improve the Professional Quality of Distinguish Hidden Trouble

C. Combined With the Related Hidden Danger Identification Method

\section{APPLICATION AND PROSPECT}

Extreme weather has a great impact on the city traffic, resulting traffic risk is increasing significantly. Therefore, it is important to identify the traffic risk point in extreme weather conditions by using scientific and reasonable method. This study will help traffic managers to recognize the traffic risk point, rapid and scientific decision and reduce casualties, economic losses and social influence caused by the extreme weather.

\section{ACKNOWLEDGMENT}

The authors would like to show great appreciation for the support from the Ministry of Industry and Information Technology of P. R. China under the Major Program of national science and technology with No. 2013ZX01045-003-002.

\section{REFERENCES}

[1] ZHAO Dong-lin, CHEN Dong-sheng. The Feasibility Study of "The Internet of Things Application Demonstration Project of Maintaining Road Traffic Unblocked in Extreme Meteorological Conditions”[R]. Beijing: Beijing Municipal Commission of Transport. 2011.

[2] SUI L Y, CHEN Z H, HE Y G. The Application Based on Internet of Things in Road Traffic Weather [C]// Proceedings of $2^{\text {nd }}$ Conference on Transportation Information and Safety, Wuhan: American Society of Civil Engineers, 2013: 309-314.

[3] WU-Jiang. Risk Prevention and Emergency Management[M]. Beijing: Dangjian Publishing House, 2011.

[4] WANG Dian-hai. Traffic Flow Theory [M]. People's Commumication Press, 2002.

[5] LIU Gong-ming. The Prevention of Road Traffic Accidents[M]. Changchun: Jilin Publishing House, 2001.

[6] WANG Li-xun, CHEN Zhi-hong, CHEN Bang-dao, LI Wei. Water logging Road Traffic Emergency Disposal Based on Internet of Things [J]. Journal of Highway and Transportation Research and Development , 2013, vlo.30 No.S1: 107-112.

[7] CHEN Zhi-hong, SUI Li-ying, ZNANG Xiao-liang. Total Framework and Operation Mechanism Research Based on Internet of Things of Maintaining Traffic Smooth in Bad Weather [J]. Journal of Highway and Transportation Research and Development , 2013, vlo.30 No.S1: 85-90.

[8] HUANG Liang. The Identification Method for Traffic Accident Potential Spots[D]. Dalian: Dalian Jiaotong University, 2009.

[9] WANG Xiao-hui, LIU Dong, CHEN Qian, et al. Study on the Early Warning Index System of City Road Traffic Safety [J]. Highway \& Automotive Applications, 2010,(137): 48-51.

[10] LIU Zhuo. The Research of Key Technologies of Traffic Safety and Risk Assessment[D]. Chengdu: Southwest Jiaotong University, 2013.

[11] ZHANG Yan, HONG Ou-bo, SUN Xiao-guang. Analysis and Solving Measures of Traffic Accident Causes of Freeway in Heavy Fog [J]. China Science and Technology Information , 2008,19: 294-297. 